\title{
The Decrease of n-3 Fatty Acid Energy Percentage in an Equicaloric Diet Fed to B6C3Fe Mice for Three Generations Elicits Obesity
}

\author{
Ingeborg Hanbauer, ${ }^{1}$ Ignacio Rivero-Covelo, ${ }^{1}$ Ekrem Maloku, ${ }^{1}$ Adam Baca, ${ }^{2}$ Qiaoyan Hu, ${ }^{1}$ \\ Joseph R. Hibbeln, ${ }^{2}$ and John M. Davis ${ }^{1}$ \\ ${ }^{1}$ Department of Psychiatry, School of Medicine University of Illinois at Chicago, 1601 W. Taylor Street, Chicago, IL 60612, USA \\ ${ }^{2}$ National Institutes on Alcohol Abuse and Alcoholism, NIH, Bethesda, MD 20892-9410, USA \\ Correspondence should be addressed to Ingeborg Hanbauer, ihanbauer@psych.uic.edu
}

Received 8 May 2009; Revised 16 June 2009; Accepted 30 June 2009

Recommended by Pietro Giusti

\begin{abstract}
Feeding mice, over 3 generations, an equicaloric diet in which $\alpha$-linolenic acid, the dietary precursor of n-3 polyunsaturated fatty acids, was substituted by linoleic acid, the dietary precursor of $n-6$ polyunsaturated fatty acids, significantly increased body weight throughout life when compared with standard diet-fed mice. Adipogenesis observed in the low $n-3$ fatty acid mice was accompanied by a 6-fold upregulation of stearyl-coenzyme A desaturase 1 (Scd1), whose activity is correlated to plasma triglyceride levels. In total liver lipid and phospholipid extracts, the sum of n-3 fatty acids and the individual longer carbon chain acids, eicosapentaenoic acid (20:5n3), docosapentaenoic acid (22:5n3), and docosahexaenoic acid (22:6n3) were significantly decreased whereas arachidonic acid (20:4n6) was significantly increased. In addition, low $n$ - 3 fatty acid-fed mice had liver steatosis, heart, and kidney hypertrophy. Hence, reducing dietary $\alpha$-linolenic acid, from 1.02 energy $\%$ to 0.16 energy $\%$ combined with raising linoleic acid intake resulted in obesity and had detrimental consequences on organ function.
\end{abstract}

Copyright (C) 2009 Ingeborg Hanbauer et al. This is an open access article distributed under the Creative Commons Attribution License, which permits unrestricted use, distribution, and reproduction in any medium, provided the original work is properly cited.

\section{Introduction}

Obesity is generally associated with a lifestyle where caloric energy intake exceeds energy expenditure. Increased fat and carbohydrate consumption creates a scenario for increased energy intake and storage of superfluous energy in adipose tissue. Pre-existing adipocytes can accumulate fatty acids as triglycerides resulting in adipose tissue expansion. On the other hand, adipose tissue mass may also be increased by de novo adipocyte differentiation [1-3]. In fact, it has been shown that nutritional or environmental changes can alter the equilibrium between adipocyte and preadipocyte populations [4]. In this context, Ailhaud et al. [1] proposed in a rodent model that excessive consumption of dietary n-6 fatty acid (FA)s with insufficient amounts of n-3 FAs might become another risk factor for obesity. To this date, little is known on the pathophysiological consequences of a disproportional intake of polyunsaturated fatty acids.
To obtain a model for "Western" diets that may still be used in many human cohorts we chose to feed mice, for at least three generation, an equicaloric diet which had disproportionate n-6 FA/n-3 FA ratio and a cholesterol level that was higher than in a vegetarian diet but below atherogenic level. Here, we report a serendipitous finding of obesity in offspring of mice fed a safflower oil-based diet (low n-3 diet, Harlan Teklad, TK 00522) over three generations in comparison to an equicaloric soy and soy oil-based diet (standard n-3, Harlan Teklad, TD 7912). Neither diet contained long chain omega- 3 nor omega- 6 fats detectable in direct compositional analysis, thus the 18 carbon precursors were the sources of essential fatty acids (Table 1 ). The low n-3 diet had lower $\alpha$-linolenic acid $(0.16$ energy \% versus 1.02 energy \%) and higher linoleic acid (12.31 energy \% versus 9.68 energy \%) compared to the standard n-3 diet. As shown in Table 1, the low n-3 diet had similar energy derived from protein and fats and had only slightly higher 


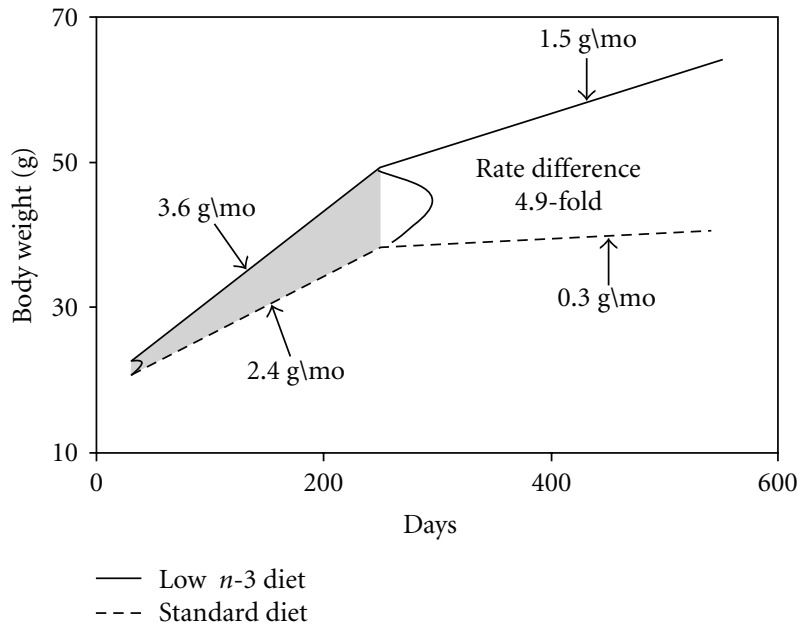

FIGURE 1: The increase of body weight over time of mice fed the low n-3 fatty acid- or standard rodent diet. A mixed regression model was calculated as described in statistical analysis section. The full line represents the rate of increase in low $\mathrm{n}-3$ fatty acid mice $(n=$ 96), and the dashed line represents the rate of increase in standard $\mathrm{n}-3$ fatty acid diet-fed mice $(n=76)$.

energy (3.4\%) from carbohydrates compared to the standard $\mathrm{n}-3$ diet. The energy percentage contributed by saturated and monounsaturated fatty acids was $21 \%$ and $28 \%$ lower in the low $\mathrm{n}-3$, compared to the standard n-3 diet.

\section{Obesity in Mice Fed a Low-Energy $\%$ n-3 FA Diet}

The body weight of the third generation of male mice on low n-3 diet was significantly higher than that of their corresponding mates on standard n-3 diet throughout their life. The low $\mathrm{n}-3$ diet group consumed $352.1 \pm 10.7 \mathrm{Kcal}$ within a 3-week period whereas mice on standard diet, for the same time period, consumed $336.6 \pm 7.1 \mathrm{Kcal}$. The body weight of both diet groups was measured biweekly, and the rate of increase in body weight was computed using a mixed regression model [5]. Figure 1 shows that low $\mathrm{n}-3$ mice $(n=$ 96) gained $3.6 \mathrm{~g} / \mathrm{mo}$ whereas control mice $(n=76)$ gained $2.4 \mathrm{~g} / \mathrm{mo}$ from time of weaning to the end of adulthood. In senescent low $\mathrm{n}-3$ mice, the rate of increase in body weight was $1.5 \mathrm{~g} /$ month compared to $0.3 \mathrm{~g} / \mathrm{mo}$ in senescent standard n-3 mice. As pointed out in Figure 1, a 4.9-fold rate difference $\left(P=7 \cdot 10^{-10}\right)$ between low $\mathrm{n}-3$ and standard $\mathrm{n}-3$ mice occurred in the senescent group (older than 240 days; open area) in contrast to a 0.5 -fold rate difference in the adult group (shaded area).

As evident from the data reported in Figure 2, mice on low $\mathrm{n}-3$ diet were prominently larger, and their body length (measured from neck to tail) was strikingly longer than in mice on standard n-3 diet (low n-3 diet: $8.26 \pm 0.11 \mathrm{~cm}(n$ $=10)$ versus standard n-3 diet: $7.8 \pm 0.14 \mathrm{~cm},(n=6) P<$ .025). Another striking difference between low n-3 FA-fed and standard n-3 mice was the increased mass of inguinalabdominal fat (Figures 2(a) and 2(b)) which suggests that
TABLE 1: Fatty acid composition of standard and low n-3 fatty acid diet expressed as energy percentage.

\begin{tabular}{|c|c|c|}
\hline & $\begin{array}{c}\text { Standard n-3 diet } \\
7912(3.1 \mathrm{Kcal} / \mathrm{g})\end{array}$ & $\begin{array}{c}\text { Low n-3 diet } \\
\text { TD } 00522(3.3 \mathrm{Kcal} / \mathrm{g})\end{array}$ \\
\hline & \multicolumn{2}{|c|}{ Energy percentage } \\
\hline Protein $\$$ & 25 & 23 \\
\hline Fats $\$$ & 17 & 16.8 \\
\hline Carbohydrates $^{\$}$ & 58 & 60 \\
\hline $10: 0$ & 0.00 & 0.00 \\
\hline $12: 0$ & 0.00 & 0.01 \\
\hline $14: 0$ & 0.00 & 0.05 \\
\hline $16: 0$ & 2.42 & 1.84 \\
\hline $18: 0$ & 0.10 & 0.07 \\
\hline $20: 0$ & 0.07 & 0.07 \\
\hline 22:0 & 0.06 & 0.05 \\
\hline $24: 0$ & 0.04 & 0.03 \\
\hline $16: 1$ & 0.02 & 0.02 \\
\hline $18: \ln 9$ & 3.91 & 2.78 \\
\hline $18: \ln 7$ & 0.16 & 0.10 \\
\hline $20: \ln 9$ & 0.07 & 0.06 \\
\hline $22: \ln 9$ & 0.02 & 0.01 \\
\hline 24:1n9 & 0.00 & 0.02 \\
\hline $18: 2 \mathrm{n} 6$ & 9.68 & 12.31 \\
\hline $18: 3 \mathrm{n} 6^{*}$ & 0.00 & 0.00 \\
\hline $20: 2 \mathrm{n} 6$ & 0.01 & 0.01 \\
\hline $20: 3 n 6^{*}$ & 0.00 & 0.00 \\
\hline $20: 4 n 6^{*}$ & 0.00 & 0.00 \\
\hline $22: 2 \mathrm{n} 6^{*}$ & 0.00 & 0.00 \\
\hline $22: 4 n 6^{*}$ & 0.00 & 0.00 \\
\hline $18: 3 n 3$ & 1.02 & 0.16 \\
\hline $20: 5 n 3^{*}$ & 0.00 & 0.00 \\
\hline $22: 6 n 3^{*}$ & 0.00 & 0.00 \\
\hline
\end{tabular}

* Fatty acid concentration is below 0.01 energy \%.

${ }^{\$}$ Values were provided by Supplier. Values for individual fatty acids represent the average of three measurements and are expressed as energy \%.

lipid metabolism was altered in low n-3 diet-fed mice. A significant increase in weight gain in the third generation of low n-3 mice was noted at 50 days of age, and the onset of inguinal obesity was observed in mice 5 months and older.

Adipose tissue is in feedback communication with the liver via adipokines, lipid factors, or lipoproteins. The adipohepatic axis, being the link between adipose tissue and liver, is important in regulating the utilization and flux of lipids in both tissues. A deregulation of either one could have detrimental effects on both tissues. In fact, as depicted in Figure 2(b) (arrow), low n-3 FA-fed mice had liver steatosis that was corroborated in Oil-red-O- stained liver sections. In the liver of low n-3 mice, a high density of macrovesicular fat droplets is notable while in a control liver slice fat droplets were absent (Figure 3).

As pointed out by Tilg and Moschen [6], adipose tissue is not just an energy storage site but it also releases adipocytederived mediators that create a link between obesity and inflammatory diseases. As soon as adipocytes get loaded 


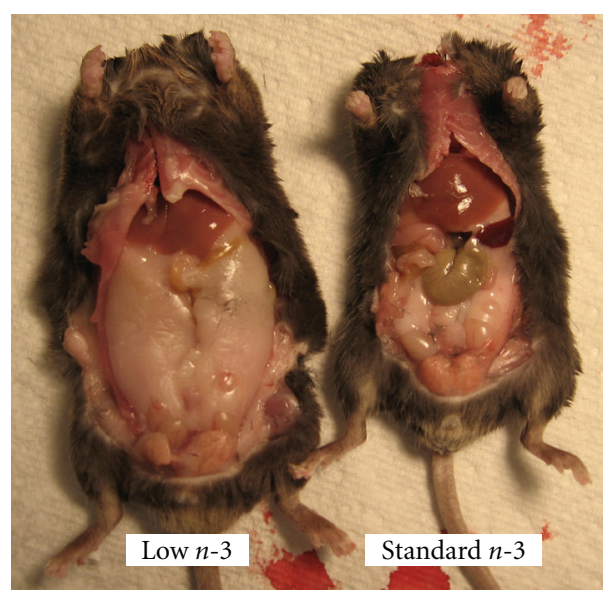

(a)

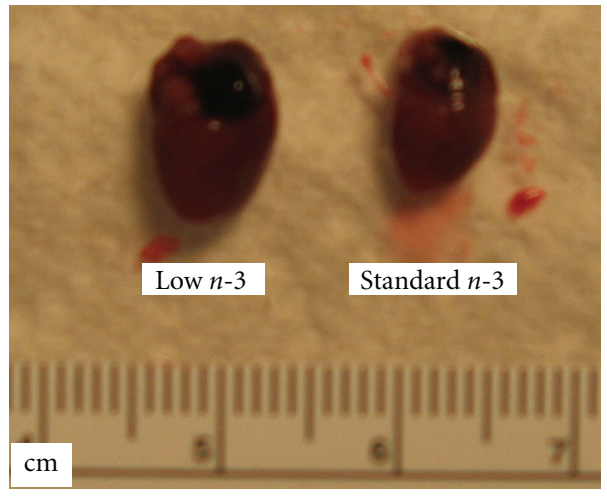

(c)

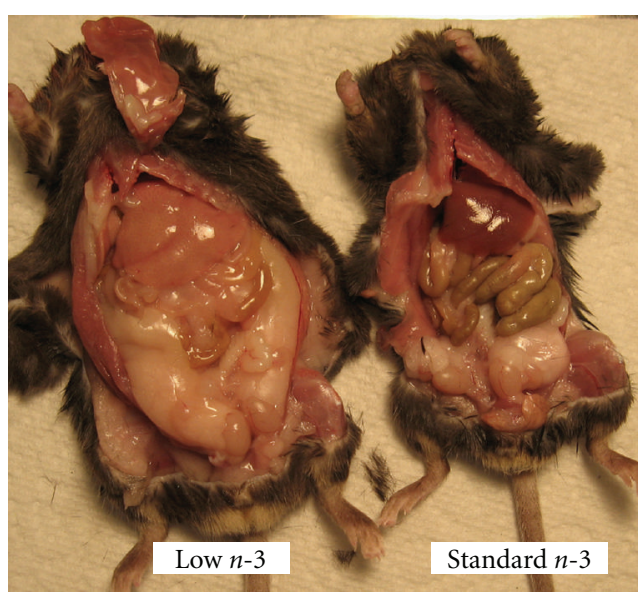

(b)

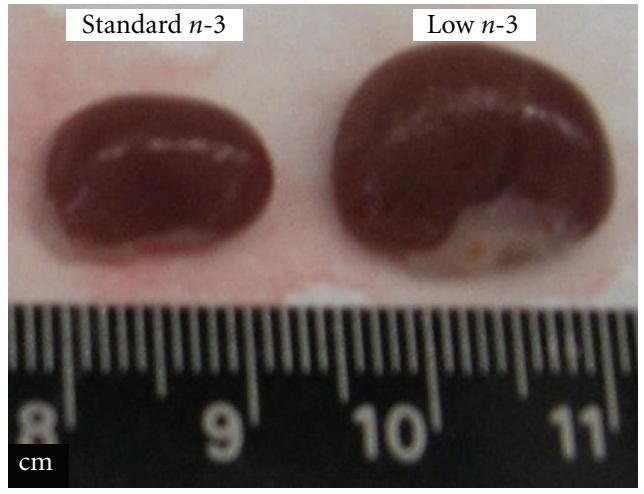

(d)

Figure 2: (a) Comparison between a low n-3 fatty acid and a standard n-3 fatty acid diet-fed mouse shows increased inguinal abdominal fat and body length. (b) Comparison of low n-3 fatty acid and a standard diet mouse shows increased body length increased inguinal abdominal fat and liver steatosis (arrow) in the low n-3 fatty acid mouse. (c) Comparison of hearts and (d) comparison of kidneys from low n-3 fatty acid- and standard n-3 fatty acid-fed mice.

TABLE 2: The effect of low n-3 diet on organ and body weight of five months old male mice. The data are expressed as mean \pm SD and statistical significance was estimated by using $t$-test, $P<.03$

\begin{tabular}{lcc}
\hline & Standard $\mathrm{n}-3 n=6$ & Low $\mathrm{n}-3 n=10$ \\
\hline Heart $(\mathrm{mg})$ & $197 \pm 28.3$ & $230 \pm 25.2^{*}$ \\
Kidney $(\mathrm{mg})$ & $433 \pm 132$ & $570 \pm 81.2^{*}$ \\
Spleen $(\mathrm{mg})$ & $141 \pm 31.1$ & $147 \pm 22.4$ \\
Body weight $(\mathrm{g})$ & $34.3 \pm 3.4$ & $47.1 \pm 4.5^{*}$ \\
\hline${ }^{*} P<.03$. & &
\end{tabular}

with triglycerides other cells such as endothelial cells, leukocytes, and macrophages invade the adipose tissue. An enhanced crosstalk between macrophages and adipocytes is thought to trigger the release of proinflammatory cytokines and chemokines that might activate a vicious cycle of proinflammatory processes: In this context, we found that in obese low n-3 FA-fed mice hearts (Figure 2(c)) and kidneys (Figure 2(d)) were hypertrophied. A comparison of a representative number of mice per diet group depicted in Table 2 shows that, indeed, organ weights of low $n-3$ mice were significantly increased. The heart hypertrophy observed in the obese low $n-3$ mice suggests that these mice might have suffered from complications accompanied by a continuous increase of cardiac work load.

\section{Changes of Fatty Acid Profile in Liver Total Lipids and Phospholipids}

In total lipid and total phospholipid extracts of livers from low n-3 FA-fed mice, the sum of n-3 FA concentrations is significantly lower than in control mice (Table 3 ). This reduction was also reflected by a decrease of individual fatty acids such as $\alpha$-linolenic acid (18:3n-3) and the longer carbon chain FAs, eicosapentaenoic acid (20:5n-3), docosapentaenoic acid (22:5n-3), and docosahexaenoic acid $(22: 6 n-3)$. In phospholipid extracts, neither the individual nor the sums of saturated, monounsaturated, linoleic acid (18:2n-6) and higher carbon chain n-6 FA levels were altered by the low n-3 FA diet (Table 3 ). Instead in total lipid extracts, linoleic acid (18:2n-6) was reduced to $4 \%$ of control level whereas arachidonic acid (20:4n-6) was significantly increased. A decrease of linoleic acid concentration in the 


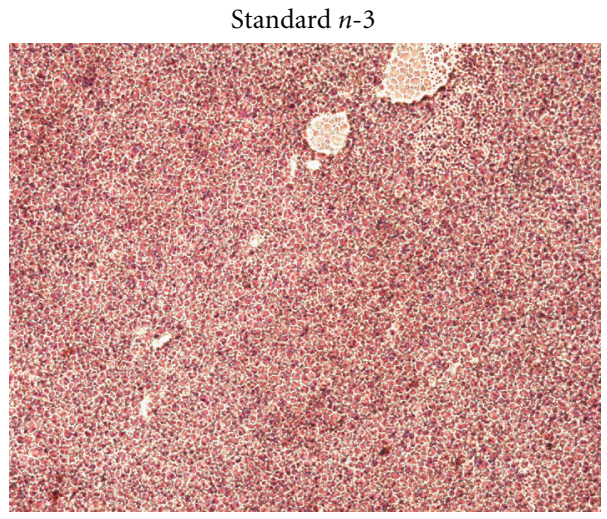

(a)

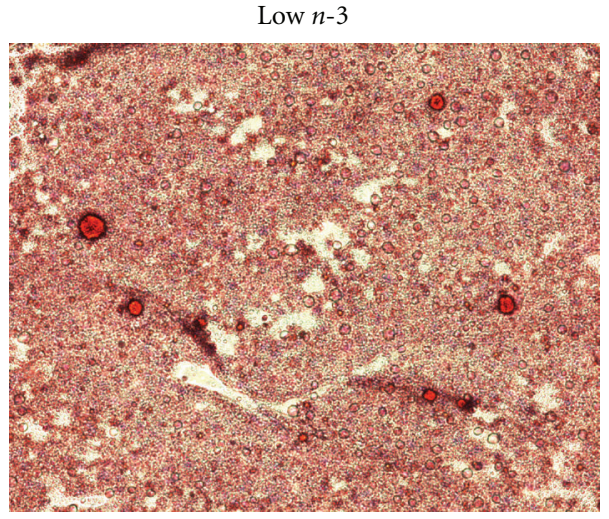

(b)

FIGURE 3: A representative liver section from standard n-3 fatty acid-fed mouse (a), low n-3 fatty acid- (b) and stained with Oil-red-O documented liver steatosis.

TABle 3: The effect of long-lasting low n-3 fatty acid diet on mouse liver fatty acid concentrations in total lipid and total phospholipid extracts. The results are expressed as mean $\pm \mathrm{SD}$ of 5 measurements per group.

\begin{tabular}{|c|c|c|c|c|}
\hline \multicolumn{5}{|c|}{ Fatty acids in total lipid and total phospholipid liver extracts ( $\mu \mathrm{g} / \mathrm{mg}$ tissue) } \\
\hline & \multicolumn{2}{|c|}{ Total Lipids } & \multicolumn{2}{|c|}{ Total Phospholipids } \\
\hline & Standard n-3 & Low n-3 FA & Standard n-3 & Low n-3 FA \\
\hline sum saturates & $15.2 \pm 3.2$ & $18.7 \pm 8.1$ & $2.72 \pm 0.7$ & $1.98 \pm 0.6$ \\
\hline sum monosats. & $12.3 \pm 7.3$ & $21.6 \pm 11.6$ & $1.24 \pm 0.22$ & $0.93 \pm 0.31$ \\
\hline $18: 2 n-6$ & $5.55 \pm 1.14$ & $0.23 \pm 0.22$ & $1.34 \pm 0.49$ & $0.97 \pm 0.36$ \\
\hline $18: 3 n-6$ & $0.088 \pm 0.02$ & $0.39 \pm 0.34$ & $0.020 \pm 0.008$ & $0.017 \pm 0.007$ \\
\hline $20: 2 n-6$ & $0.17 \pm 0.046$ & $0.66 \pm 0.61$ & $0.041 \pm 0.018$ & $0.030 \pm 0.015$ \\
\hline $20: 3 n-6$ & $0.70 \pm 0.11$ & $1.16 \pm 0.70$ & $0.17 \pm 0.059$ & $0.13 \pm 0.063$ \\
\hline $20: 4 n-6$ & $5.54 \pm 0.38$ & $6.64 \pm 1.0$ & $1.64 \pm 0.55$ & $1.36 \pm 0.61$ \\
\hline $22: 2 n-6$ & $0.13 \pm 0.008$ & $0.077 \pm 0.065$ & $0.00 \pm 0.00^{\pi}$ & $0.00 \pm 0.00^{\pi}$ \\
\hline $22: 4 n-6$ & $0.18 \pm 0.05$ & $0.68 \pm 0.48$ & $0.033 \pm 0.016$ & $0.037 \pm 0.021$ \\
\hline $18: 3 n-3$ & $0.087 \pm 0.037$ & $0.068 \pm 0.061$ & $0.01 \pm 0.004$ & $0.00 \pm 0.00^{*}$ \\
\hline $20: 3 n-3$ & $0.012 \pm 0.007$ & $0.011 \pm 0.013$ & $0.002 \pm 0.001$ & $0.001 \pm 0.001$ \\
\hline $20: 5 n-3$ & $0.097 \pm 0.013$ & $0.027 \pm 0.015^{*}$ & $0.022 \pm 0.0014$ & $0.00 \pm 0.00^{*}$ \\
\hline $22: 5 n-3$ & $0.17 \pm 0.04$ & $0.055 \pm 0.025^{*}$ & $0.037 \pm 0.017$ & $0.01 \pm 0.004^{*}$ \\
\hline $22: 6 n-3$ & $2.37 \pm 0.27$ & $0.78 \pm 0.093^{*}$ & $0.73 \pm 0.34$ & $0.24 \pm 0.08^{*}$ \\
\hline sum n-6 ${ }^{v}$ & $6.39 \pm 0.47$ & $9.22 \pm 2.75$ & $1.89 \pm 0.63$ & $1.55 \pm 0.68$ \\
\hline $\operatorname{sum} \mathbf{n}-3^{v}$ & $2.65 \pm 0.26$ & $0.88 \pm 0.14^{*}$ & $0.80 \pm 0.37$ & $0.25 \pm 0.085^{*}$ \\
\hline$\%$ n-6 HUFA & $70.7 \pm 2.55$ & $91.1 \pm 1.28^{*}$ & $70.3 \pm 8.6$ & $84.7 \pm 6.5^{*}$ \\
\hline
\end{tabular}

${ }^{\nu}$ Sum n-6 and sum n-3 include the critically bioactive 20 and 22 carbon fatty acids only. The n-6 \% HUFA is calculated as: (sum n-6)/[(sum n-6)/ (sum n-3)]. 100.

${ }^{\pi}$ Concentrations are lower than 0.01 . The statistical significance was calculated using an independent samples T-test as described in methods (Sig. 2-tailed: $P<0.05)$.

${ }^{*} P<0.05$

total liver lipid extract elicited by low n-3 FA diet was also reported by other researchers [7].

Long lasting deprivation of n-3 FA marginally increased the sum of monounsaturated FAs in total liver lipids (Table 3). Since we observed also a tentative increase of individual monounsaturated FAs (data not shown), we studied Scd1, a gene encoding liver stearoyl-coenzyme A desaturase1. Elevation of hepatic stearoyl-coenzyme A desaturase 1 activity is correlated to increased plasma triglyceride levels [8]. Dietary and hormonal factors were shown to upregulate liver desaturase activity whereas polyunsaturated FAs were shown to repress desaturase gene upregulation [9]. As shown in Table 4, long lasting low n-3 FA diet elicited a 6.5-fold upregulation of liver stearyl-coenzyme A desaturase 1 ( $\mathrm{Scd} 1$ ) gene while $\mathrm{Scd} 2$ remained unchanged. Increased SCD1 activity was shown to be rate-limiting in the formation of triglyceride and cholesterol esters [10]. More studies are needed to understand whether liver Scd1 gene was upregulated because n-3 FA levels were too low to repress it or other diet-related changes participated in a more complex 
TABle 4: The effect of low n-3 fatty acid diet on stearyl-coenzyme A desaturase 1 (Scd1) and stearyl-coenzyme A desaturase 2 (Scd2) gene regulation in mouse liver. Rt-PCR was performed on total RNA extracts from mouse livers (SABiosciences, Frederick, MD). $\mathrm{Ct}$ values corrected against house keeping gene, beta actin, are expressed as average of 4 measurements per group.

\begin{tabular}{lccc}
\hline Gene & $\Delta$ Ct Standard n-3 & $\Delta$ Ct Low n-3 & Fold Change \\
\hline Scd1 & -2.55 & -5.24 & 6.46 \\
Scd2 & 10.98 & 10.83 & 1.1 \\
\hline
\end{tabular}

gene upregulation. Since monounsaturated FAs are ratelimiting substrates for lipid synthesis in the liver [10], it can be inferred that, the tentative increase of monounsaturated FAs in total lipid extracts shown in Table 3 might be linked to lipogenesis.

\section{Discussion}

In the last decade, research on obesity revealed a redundancy of complex networks involved in the regulation of energy homeostasis. This multitude of regulatory pathways appears to serve the purpose to maintain energy demand during fasting and to store excessive energy during extensive food consumption. The present paper shows that long-lasting n-3 FA deficiency accompanied with n-6 FA excess affects liver function in a manner that may consecutively lead to obesity. One indication for altered energy efficiency set points elicited by long-lasting n-3 FA deficiency is the increase of liver triglyceride synthesis as indicated by fatty liver shown in Figure 2, macrosteatosis shown in Figure 3, and the upregulation of (Scd1), a member of the stearyl-CoA desaturase family shown in Table 4.

Reports in literature showed that hepatic lipogenesis is increased during high carbohydrate or high fat diet $[11,12]$ but failed to occur in mice with a targeted deletion of SCD1. Since increased SCD1 expression results in increased monounsaturated FA synthesis the tentative increase of the sum of monounsaturated FAs shown in Table 3 may be another indication of increased hepatic lipogenesis. Recently, it was shown that the degree of SCD1 activation correlates with increased plasma triglyceride levels in humans and mice [10].

The novel finding of the present paper documents that SCD1 is upregulated by an increased n-6/n-3 FA ratio diet, in contrast to high fat or low fat combined with high carbohydrate diet. Under this circumstance, the downstream events of lipogenesis could be upregulated by decreased n3 polyunsaturated FA and increased n- 6 polyunsaturated FA levels. Hence, in low n-3 mice, the deficit of n-3 FAs, particularly the high carbon chain group (Table 3 ), might be responsible for the lack of suppression of lipogenic genes. This is in line with findings showing that n-3 polyunsaturated FAs play an important role in the regulation of lipogenic genes in abdominal fat and brain [13]. Extrapolation of the present data to human populations showed a stark parallel to the increased dietary intake of n-6 FAs in most developed countries in the last 100 years. Due to the competitive relationship between n-3 and n-6 FAs the inevitable increase of the n- 6 HUFA pool may irreversibly lead to both obesity and the inflammation resulting in increased mortality $[14,15]$. Several epidemiological studies in human cohorts showed that a diet rich in n-6 FA and poor in n-3 FA altered the FA profile in plasma lipids $[16,17]$. The outcome of these studies focusing on human cohorts showed that a high-ratio n-6/n-3 FA diet over many generations may have triggered gradual and progressive changes in brain function that could heighten despair or suboptimal social interaction and thus increase the risk of personality disorders [18]. In fact, an increase in severity of acute mania [19] or depressive disorders in elderly [17] were shown to be inversely related to low plasma levels of n-3 polyunsaturated fatty acids. Ongoing research in this laboratory is directed to study the long-term effect of different n-6/n-3 FA ratios in regard to pathophysiological and/or behavioral changes in mice.

\section{Materials and Methods}

5.1. Animals. Animals came from our heterozygous mice breeding colony $\mathrm{B} 6 \mathrm{C} 3 \mathrm{Fe}$ a/a- $\mathrm{R} \mathrm{n}^{\mathrm{rl}} / \mathrm{J}$ (originally obtained from Jackson Laboratories, Bar Harbor, ME, USA). Backcross $\mathrm{B} 6 \mathrm{C} 3 \mathrm{Fe}$ male mice were bred in house by pairing male wild type B6C3Fe mice with wild type B6C3Fe dams. The founder strain is not obesity-prone and has no known vulnerabilities to dietary changes in polyunsaturated fatty acids (The Jackson Laboratory Mice Database). Two to five male offspring were housed per cage and had access to their respective diet and water ad libitum. One group of the breeder pairs received standard rodent diet (Harlan Teklad $7012,3.1 \mathrm{Kcal} / \mathrm{g}$ ) and is also referred to as standard n-3 diet. The second group was fed low n-3 diet throughout three consecutive generations (Harlan Teklad TD.00522, $3.3 \mathrm{Kcal} / \mathrm{g}$, with an addition of $750 \mathrm{ppm}$ cholesterol, as specified by the supplier). This cholesterol concentration is above the range present in "vegetarian" diets $(0-280 \mathrm{ppm})$ but is below the range that is considered atherogenic (2000 ppm) for susceptible mouse models [20].

Pellets from each diet were extracted by a modification of the Folch method [21] using 23 : 0 PC as internal standard. Chloroform extracts were dried under $\mathrm{N}_{2}$ and reconstituted in $500 \mu \mathrm{L}$ of $100: 3: 0.3$ hexane : MTB : acetic acid. FAs were transmethylated with $14 \% \mathrm{BF}_{3}-\mathrm{MeOH}$ at $100^{\circ} \mathrm{C}$ for 60 minutes and methyl esters were analyzed by gas chromatography as described by Morrison and Smith [22].

The body weight of both diet groups was measured biweekly. In the present study, male wild type mice were used between the ages of two to eight months for biochemical tests. All animal procedures were approved by the University of Illinois at Chicago Animal Care Committee.

5.2. Histological Analysis. Livers were quickly excised from carbon dioxide-euthanized mice and frozen on dry ice. Frozen livers were used to prepare sections $(5 \mu \mathrm{m})$ that 
were fixed in $10 \%$ neutral buffered formalin (10 minutes). After several washes the sections were stained with Oil-Red O for 10 minutes, washed several times with water, and counterstained with hematoxylin for 2 minutes. The sections were mounted with coverslips, observed under a microscope, and photographed.

5.3. Liver Fatty Acid Analysis by Gas Chromatography. Sevenmonth-old mice were decapitated; their livers were rapidly removed and frozen on dry ice. The frozen tissues were subjected to total lipid extraction by a modification of the Folch method using 23:0 PC as internal standard. Chloroform extract was dried down under $\mathrm{N}_{2}$ and reconstituted in hexane:MTB:acetic acid (100: $3: 0.3)$ for solid phase extraction to separate lipid classes. The total lipid and phospholipids extracts were transmethylated with 14\% BF3-methanol and the methyl esters analyzed by gas chromatography as previously described [23].

5.4. Liver RNA Isolation and Real-Time PCR. Total RNA was extracted with Trizol (Invitrogen) according to the protocol of the manufacturer. The obtained total RNA pellets were dissolved in RNAse-free water, and possible contaminating DNA was removed using the DNA-free kit (Ambion). The A260/A280 was measured spectrophotometrically.

The regulation of Scd1 and Scd2 gene expression was measured by using SYBR green-based real time PCR in liver RNA samples. RNA extraction and quantitative real time PCR (qRT-PCR) were performed at the SABiosciences Service Core, Frederic, MD, based on SABiosciences' ArrayGrade Total RNA Isolation Kit (GA-013), RT2 qPCR primer assays (PPM05664E (scd1), PPM32741A (scd2), and PPM02945A (beta actin)) and RT2 qPCR master mix (PA-011). The mRNA expression level for each gene was normalized against the expression level of the house-keeping gene beta actin within each sample by the comparative $\mathrm{Ct}$ $(\Delta \mathrm{Ct})$ method. The fold-change values were calculated using the $\Delta \Delta \mathrm{Ct}$ method.

5.5. Statistical Analysis. Results are expressed as mean $\pm \mathrm{SD}$, statistical significance was calculated using Student $t$ test for independent samples (SPSS v.15 for Windows), the Levene's Test was used to measure the Equality of Variances, and differences were considered statistically significant at $P<.05$.

The rate of body weight increase was computed using a linear mixed regression model [5]. The slope obtained for each individual mouse was fitted in a linear regression model and then fitted in a mixed regression model of two linear slopes from 30 to 250 days and 250 to 550 days. The model is Body weight $=\beta 0+\beta 1$ Diet $+\beta 2$ Time $+\beta 3$ Young versus Old $+\beta 4$ Diet ${ }^{*}$ Time $+\beta 5$ Time* Age $(\beta 0=$ intercept of SRD group from 30 to 250 days; $\beta 1=$ intercept difference of two groups; $\beta 2=$ slope of SRD group from 30 to 250 days; $\beta 3=$ intercept difference of SRD group from 250 to 550 day and 30 to 250 days; $\beta 4=$ slope difference between 2 groups; $\beta 5=$ slope difference from 250 to 550 day and 30 to 250 day).

\section{Acknowledgments}

The authors thank Drs. E. Costa and A. Guidotti for many discussions and M. S. Doueiri for technical assistance. This work was supported, in part, by NIMH Grants MH070855 to Erminio Costa and MH070855 to Alessandro Guidotti.

\section{References}

[1] G. Ailhaud, P. Guesnet, and S. C. Cunnane, "An emerging risk factor for obesity: does disequilibrium of polyunsaturated fatty acid metabolism contribute to excessive adipose tissue development?" British Journal of Nutrition, vol. 100, no. 3, pp. 461-470, 2008.

[2] P. Wang, E. Mariman, J. Renes, and J. Keijer, "The secretory function of adipocytes in the physiology of white adipose tissue," Journal of Cellular Physiology, vol. 216, no. 1, pp. 3-13, 2008.

[3] A. D. Attie and P. E. Scherer, "Adipocyte metabolism and obesity," Journal of Lipid Research, vol. 50, supplement, pp. S395-S399, 2009.

[4] I. M. Faust, P. R. Johnson, J. S. Stern, and J. Hirsch, "Dietinduced adipocyte number increases in adult rats: a new model of obesity," American Journal of Physiology, vol. 235, pp. E279-E286, 1978.

[5] D. Hedeker and R. Gibbons, Longitudinal Data Analysis, John Wiley \& Sons, New York, NY, USA, 2006.

[6] H. Tilg and A. R. Moschen, "Adipocytokines: mediators linking adipose tissue, inflammation and immunity," Nature Reviews Immunology, vol. 6, no. 10, pp. 772-783, 2006.

[7] S.-Y. Lim, J. D. Doherty, and N. Salem Jr., "Lead exposure and (n-3) fatty acid deficiency during rat neonatal development alter liver, plasma, and brain polyunsaturated fatty acid composition," Journal of Nutrition, vol. 135, no. 5, pp. 10271033, 2005.

[8] A. D. Attie, R. M. Krauss, M. P. Gray-Keller, et al., "Relationship between steaoryl CoA desaturase activity and plasma triglycerides in human and mouse hypertriglyceridemia," Journal of Lipid Research, vol. 43, pp. 1899-1907, 2002.

[9] M. T. Flowers and J. M. Ntambi, "Role of stearoyl-coenzyme A desaturase in regulating lipid metabolism," Current Opinion in Lipidology, vol. 19, no. 3, pp. 248-256, 2008.

[10] M. T. Flowers and J. M. Ntambi, "Stearoyl-coenzyme A desaturase and its relation to high-carbohydrate diets and obesity," Biochimica et Biophysica Acta, vol. 1791, pp. 85-91, 2009.

[11] L. C. Hudgins, M. Hellerstein, C. Seidman, R. Neese, J. Diakun, and J. Hirsch, "Human fatty acid synthesis is stimulated by a eucaloric low fat, high carbohydrate diet," Journal of Clinical Investigation, vol. 97, no. 9, pp. 2081-2091, 1996.

[12] W. C. Willett, "Dietary fat plays a major role in obesity," Obesity Reviews, vol. 3, pp. 59-68, 2002.

[13] H. Sampath and J. M. Ntambi, "Polyunsaturated fatty acid regulation of genes of lipid metabolism," Annual Review of Nutrition, vol. 25, pp. 317-340, 2005.

[14] W. Lands, "How is tissue fatty acid composition maintained? "Seminar to the Polyunsaturated Lipid Function Special Interest Group, December 2003, Google.

[15] C. L. Ogden, M. D. Carroll, L. R. Curtin, M. A. McDowell, C. J. Tabak, and K. M. Flegal, "Prevalence of overweight and obesity in the United States," American Medical Association, vol. 295, pp. 1549-1555, 2006. 
[16] J. R. Hibbeln, L. R. G. Nieminen, T. L. Blasbalg, J. A. Riggs, and W. E. M. Lands, "Healthy intakes of n-3 and n-6 fatty acids: estimations considering worldwide diversity," American Medical Association, vol. 83, pp. 2483S-1493S, 2006.

[17] C. Féart, E. Peuchant, L. Letenneur, et al., "Plasma eicosapentaenoic acid is inversely associated with severity of depressive symptomatology in the elderly: data from the Bordeaux sample of the three-city study," American Journal of Clinical Nutrition, vol. 87, no. 5, pp. 1156-1162, 2008.

[18] J. R. Hibbeln, "From homicide to happiness-a commentary on omega-3 fatty acids in human society," in Nutrition and Health, vol. 19, pp. 9-19, 2007.

[19] M. E. Sublette, F. Bosetti, J. C. DeMar, et al., "Plasma free polyunsaturated fatty acid levels are associated with symptom severity in acute mania," Bipolar Disorders, vol. 9, no. 7, pp. 759-765, 2007.

[20] F. Calon, G. P. Lim, F. Yang, et al., "Docosahexaenoic acid protects from dendritic pathology in an Alzheimer's disease mouse model," Neuron, vol. 43, no. 5, pp. 633-645, 2004.

[21] J. Folch, M. Lees, and G. H. Sloane-Stanley, "A simple method for the isolation and purification of total lipids from animal tissues," Journal of Biological Chemistry, vol. 226, pp. 497-509, 1957.

[22] W. R. Morrison and L. M. Smith, "Preparation of fatty acid methyl esters and dimethylacetals from lipids with boronfluoride-methanol," Journal of Lipid Research, vol. 5, pp. 600608, 1959.

[23] N. Salem Jr., M. Reyzer, and J. Karanian, "Losses of arachidonic acid in rat liver after alcohol inhalation," Lipids, vol. 31, supplement, pp. S153-S156, 1996. 


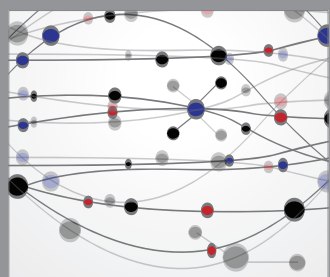

The Scientific World Journal
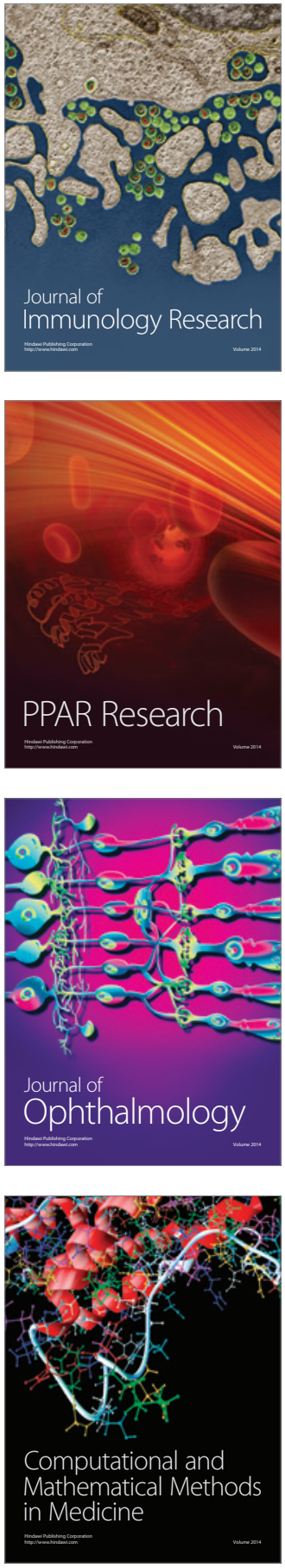

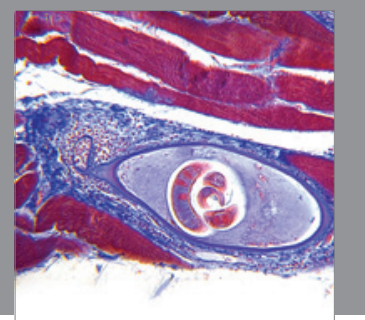

Gastroenterology

Research and Practice
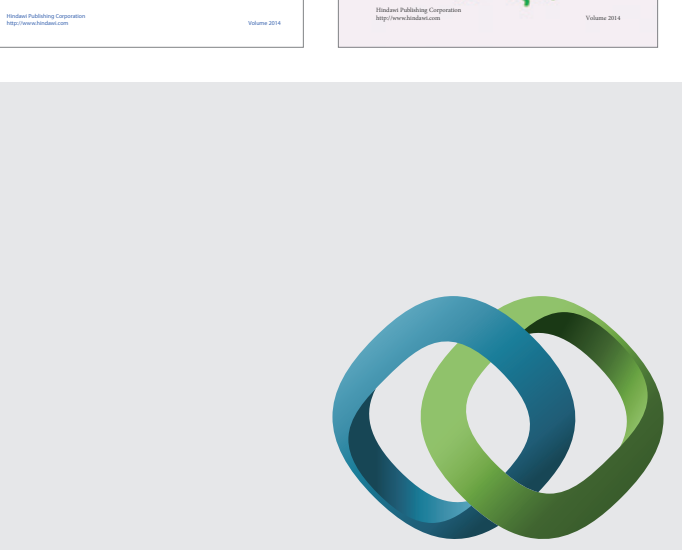

\section{Hindawi}

Submit your manuscripts at

http://www.hindawi.com
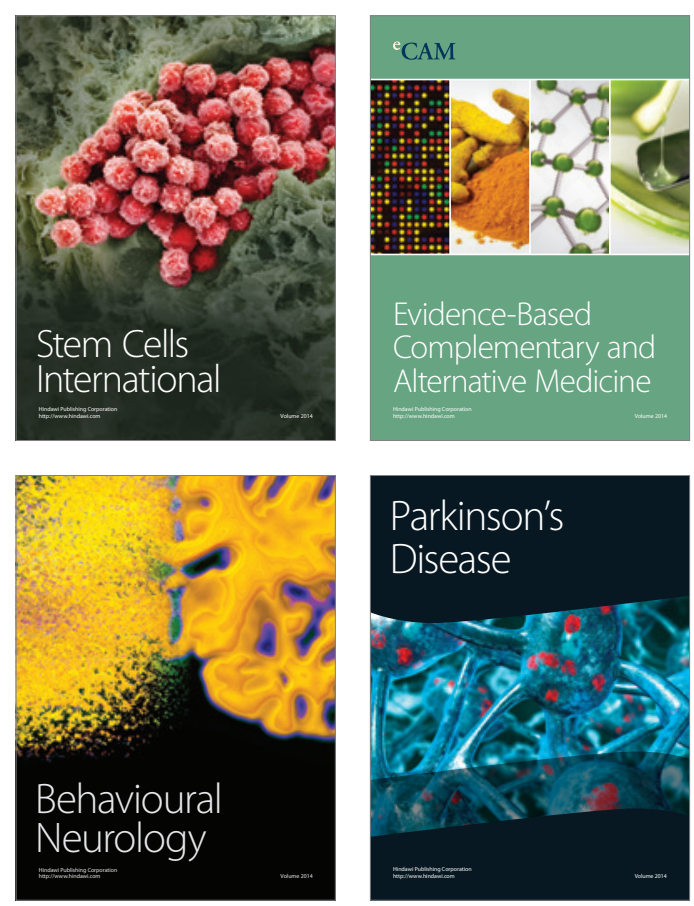

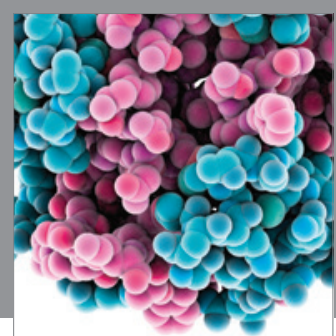

Journal of
Diabetes Research

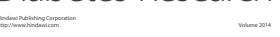

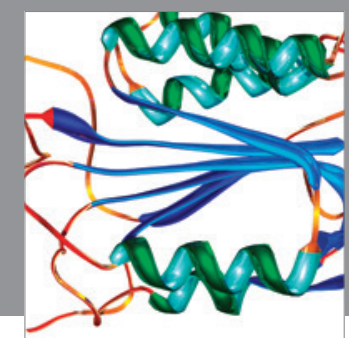

Disease Markers
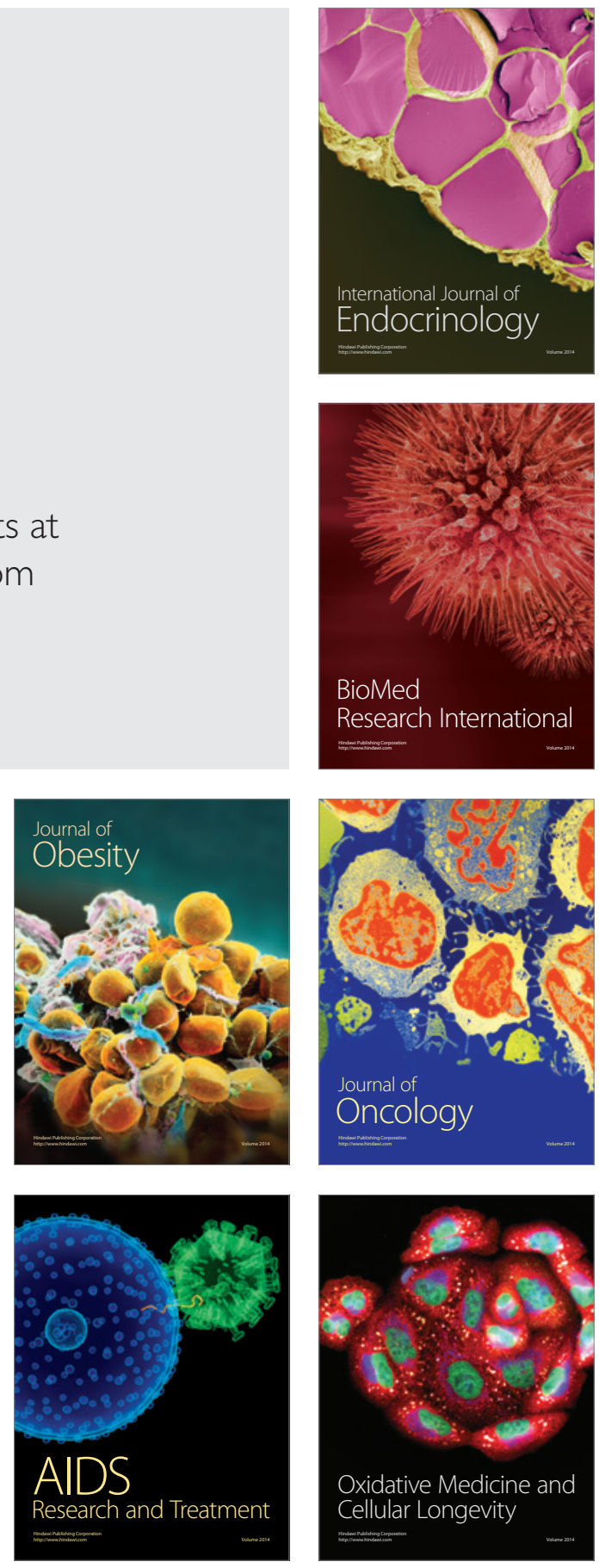